\title{
From quantum mechanics to force fields: new methodologies for the classical simulation of complex systems
}

\author{
Jean-Philip Piquemal · Kenneth D. Jordan
}

Published online: 31 March 2012

(C) Springer-Verlag 2012

Molecular dynamics simulations have become one of the key methods for simulating the properties of biological systems and complex materials. Underlying such simulations are the force fields that describe how the energy varies as a function of geometry. As a result, the reliability of the simulations depends on the quality of the force field. In many cases, this requires the explicit incorporation of polarization. For these reasons, accurate electronic structure methods are playing an increasingly important role in the parameterization of force fields.

This special issue of Theoretical Chemistry Accounts illustrates recent trends in the design and applications of accurate force fields. Of course, the field is vast and is growing rapidly; thus, we cannot claim completeness within such a collection. However, the following 15 papers reflect the present questions including the strategies for (i) the inclusion of the polarization energy and (ii) an optimal parametrization of model and highlight the directions to follow as new exciting fields of application emerge.

Published as part of the special collection of articles: From quantum mechanics to force fields: new methodologies for the classical simulation of complex systems.

\section{J.-P. Piquemal $(\bowtie)$}

UPMC, Paris 6, Laboratoire de Chimie Théorique and CNRS,

Sorbonne Université, Campus Jussieu,

Tour 12-13, 4ème étage, CC 137, 4 Place Jussieu,

UMR 7616, 75252 Paris Cedex 05, France

e-mail: jpp@1ct.jussieu.fr

K. D. Jordan $(\square)$

Department of Chemistry, University of Pittsburgh,

219 Parkman Avenue, Pittsburgh, PA 15260, USA

e-mail: jordan@pitt.edu
Three papers discuss the optimization and parametrization of new models.

Markutsya et al. present a coarse-grained model for $\beta$-Dglucose based on force matching.

$\mathrm{Wu}$ et al. propose an automation process for the AMOEBA polarizable force field parameterization of small molecules.

Pinnick et al. detailed an approach enabling fast convergence of $a b$ initio free energy perturbation calculations with the adaptive force matching method.

Three papers put in perspectives the actual importance of the polarization energy.

Kumar et al. discussed the polarizing forces of water. Guàrdia et al. detailed the importance of the polarization damping and its consequences on ion solvation dynamics.

Söderjelm focuses on polarization effects in proteinligand calculations and shows that it could extend farther than the actual induction energy.

Five papers review or propose new models explicitly incorporation polarization.

Han et al. present a new optimization of the explicit polarization X-Pol potential using a hybrid density functional.

Giese et al. illustrate the grand challenges in density functional expansion methods.

Nagata et al. propose new strategies to obtain analytic gradients in order to perform molecular dynamics simulations using the fragment molecular orbital (FMO) method with effective potentials.

Bauer et al. review recent applications and developments of the charge equilibration force fields. 
Mills et al. illustrate their new polarizable multipolar electrostatics approach based on the Kriging machine learning method and apply it to alanine.

Finally, four papers present models that are applied to difficult systems and fields of application.

Canongia Lopes et al. propose a generic and systematic force field for ionic liquids modeling.

Wang et al. described a new polarizable model capturing the energetics and dynamics of carbon monoxide.

Marjolin et al. extend polarizable models to the accurate treatment of lanthanide and actinides in solution allowing the computation of hydration free energies.
Salanne et al. discussed the inclusion of many-body effects in models for ionic liquids.

While the development of new models will continue, it is clear that electronic structure calculations and advanced parametrization strategies will play a key role in force field design for years to come. We hope that the 15 papers of this Special Issue will be of lasting values. 